\title{
A COMPUTER-ASSISTED INTERNAL EXAMINATION TRAINING SYSTEM USING BOTH ANATOMICAL AND VIRTUAL MODELS
}

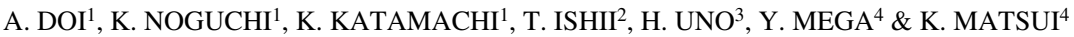 \\ ${ }^{1}$ Iwate Prefectural University, Japan. \\ ${ }^{2}$ The Japanese Red Cross Hokkaido College of Nursing, Japan. \\ ${ }^{3}$ KOKEN Co., Ltd., Japan. \\ ${ }^{4} \mathrm{JFP}$, Inc., Japan.
}

\begin{abstract}
For obstetricians and midwives, 'internal examination' refers to an important diagnostic technique in which the progress of labor is examined using the index and middle fingers inserted into the vagina or rectum. Training of this internal examination technique has been commonly performed using a model of the human body (manikin). However, with this method, it was impossible to determine visually where and how the examining fingers are touching, making it difficult for trainers to teach advanced examination skills efficiently and evaluate training achievements. Against this background, we have developed a training system for internal examination that enables simulation of normal and abnormal conditions of labor by detecting the position and direction of the examining fingers in real-time via tactile and visual perceptions using anatomical and virtual models. This system allows trainees to experience both normal and abnormal fetal descent into the pelvis. In addition to support the abnormal conditions, we propose the unique measurements function using magnetic sensors, in order to estimate the precise baby status, such as a gap of the uterine ostium or baby head. We conducted a survey of eight students at our university, and made inquiries for our system, and evaluated it for understanding where the position of ischiatic thor (spinal ischium), baby head, and uterine ostium. The result of the several questions shows that the understanding of all students is improved by using our system.

Keywords: a training system, internal examination, magnetic sensor, manikin, virtual models, virtual reality.
\end{abstract}

\section{INTRODUCTION}

Previously developed training systems for internal examination include our own system [1-3], ePelvis [4-6] developed by a group from Stanford University, and the peripartum diagnosis/delivery assistance training system [7], and the SIMone Childbirth Birthing Simulator/Manikin [8]. The ePelvis is a prototype pelvic simulator, and it attaches several sensors inside the mother's body (manikin). The peripartum diagnosis/delivery assistance training system is basically a visual learning system using video images, and is suitable for teaching and explanation but not for training of the internal examination itself. The SIMone is a model of a female abdomen with a vulva and the spinal ischium as landmarks. Inside the simulator is a fetal head, and the 19" screen monitor shows the position and approach of the head. The ePelvis, the peripartum diagnosis/delivery assistance training system, and the SIMone are not suitable for close monitoring of fingers and the evaluation of examination techniques. Our approach in our training systems described in [1-3] is different in comparison with the three systems, ePelvis, the peripartum diagnosis/delivery assistance training system, and the SIMone. We utilize magnetic sensors that are attached with two fingers, and monitor the motions of two fingers in internal examination. Our previous system was used to simulate the normal labor condition and thus was not suitable for simulating various abnormalities that can occur during labor. We thus developed a system based on fetal models that could reproduce various abnormal labor conditions (frank breech presentation, complete breech presentation, placenta previa, and face presentation) [9]. Our newly developed internal examination training system consists of models of maternal body parts, including the vagina and the uterine ostium, and fetal body parts (anatomical models), a personal computer (PC), and a magnetic sensor (Fig. 1).

(C) 2010 WIT Press, www.witpress.com

ISSN: 1755-7437 (paper format), ISSN: 1755-7445 (online), http://journals.witpress.com DOI: $10.2495 / \mathrm{DNE}-\mathrm{V} 5-\mathrm{N} 2-108-121$ 


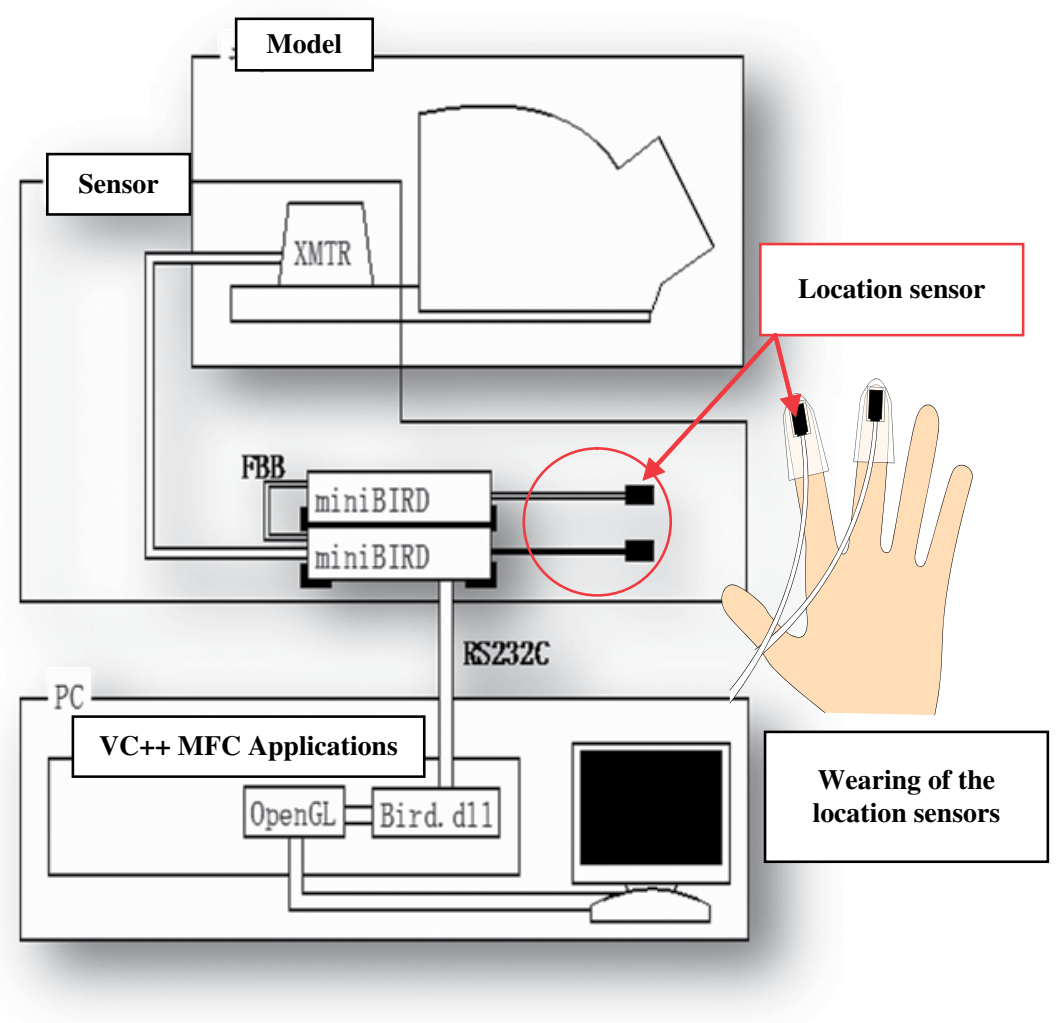

Figure 1: System configuration.

The tracking system consists of a transmitter (XMTR), a location sensor, and a controller (miniBIRD, Ascension Technology Corp.) [10-11]. Magnetic information transmitted from the transmitter is detected by the location sensor, and the location information is received by the PC. The location sensor is attached and fixed to a fingerstall made of silicon rubber, which is worn on the index and middle fingers, with which internal examination is performed. The accuracy of position and angle in miniBIRD are $1.8 \mathrm{~mm}$ RMS and $0.5^{\circ} \mathrm{RMS}$. The update rate is up to 120 measurements per second. The sensor size (OD) of our miniBIRD is $5 \mathrm{~mm}$ (Model 500).

\section{SYSTEM OVERVIEW}

Figure 2 shows the external appearance of the training system. The system supports normal labor conditions of one-finger dilatation, two-finger dilatation, full dilatation and type $\mathrm{C}$ (another type of full dilatation) and abnormal labor conditions of frank breech presentation, complete breech presentation, placenta previa and face presentation. Other conditions can be added if necessary. The monitor displays geometric models (i.e. virtual models of human body, examining fingers, etc.) of the anatomical models. An advantage of the system is that it can display on the monitor the position and the direction of the examining fingers during internal examination via the magnetic sensors worn on the examining fingers. The teacher check the motion of trainers for internal examination in the monitor, and trainers can confirm their position of fingers, too. The system thus allows trainers to evaluate visually the skill level and accuracy of examination techniques on the monitor.

Inside the human body model shown in Fig. 3 is a guiding structure to install models of the pelvis and the fetus. The left top picture shows overview, and the right top picture shows the skin of vinyl 

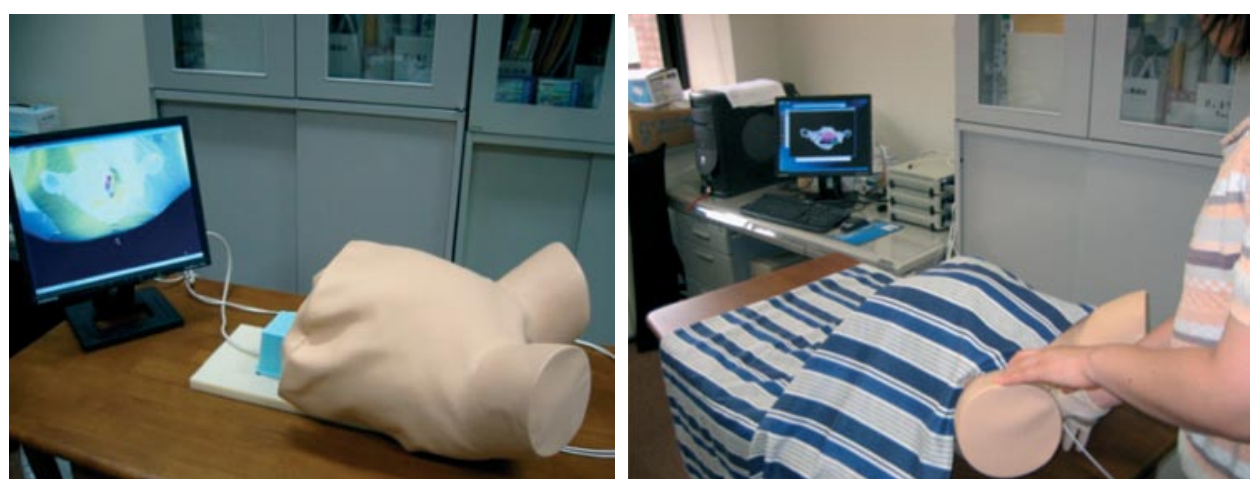

Figure 2: Our internal examination training system.
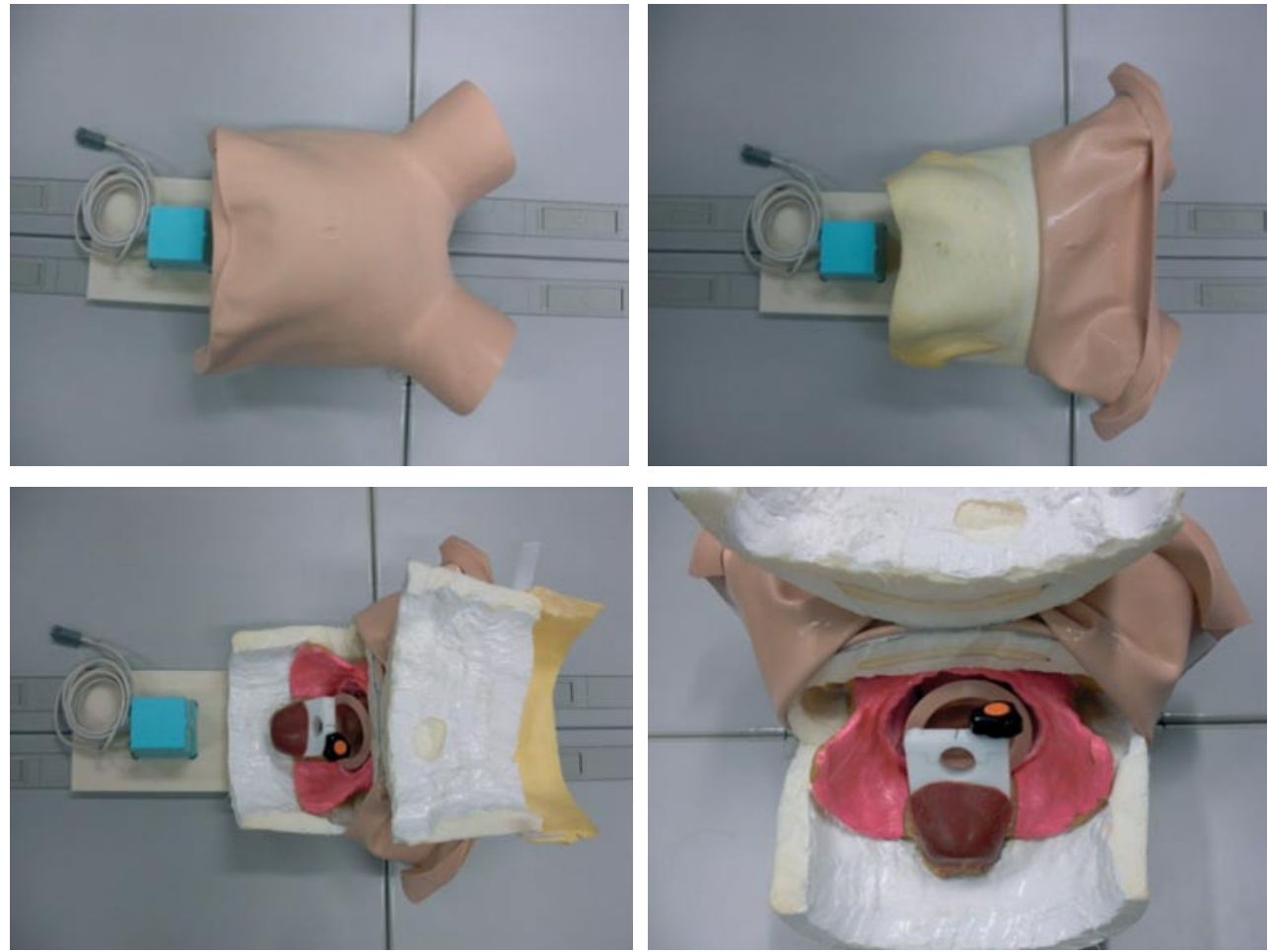

Figure 3: Overview inside the internal examination training system (left top: overview, right top: the skin of vinyl cloth and the body of foamed styrol, left bottom: the bone and guiding structure inside of the body, right bottom: the magnification of the guiding structure).

cloth and the body of foamed styrol. The left bottom picture shows the bone and guiding structure inside of the body, and the right bottom picture shows the detail of the guiding structure.

An appropriate anatomical model of the fetus should be placed in the human body model before starting training. The skin in the virtual model was created by measuring the skin with a noncontact 3D digitizer, and converting to polygonal shape. 
The entire fetal models are created by a computer aided design (CAD) software. All generated polygonal models are combined, and both color and optical information are added. The anatomical models of the pelvis and the body were created as follows: a 3D image of the pelvis was taken with a 3D-computed tomography (CT) system, the 3D image was appropriately smoothed and iso-surfaced, and the surface shape of the pelvis and the body was created. The anatomical model of the pelvis and the fetus was made by rapid proto-typing device (3D printer). The skin of mother's body with enclosing the pelvis was created by plastic material and vinyl cloth.

\section{OUR TRAINING METHOD}

Eight anatomical models of the fetus, including 4 normal and 4 abnormal models, were prepared. Figure 4 shows both 4 normal cases (left top: type $\mathrm{C}$ (near full dilatation), right top: full dilatation, left bottom: two-finger dilatation, right bottom: one-finger dilatation) and 4 abnormal cases (left top: incomplete foot presentation, right top: complete breech presentation, left bottom: placenta previa, right bottom: face presentation), respectively. These models describe only the parts that are touched by the examining fingers, and are displayed together with the whole image of the fetus.

After a fetal model was placed in the system, the corresponding virtual model is selected on the dialog on the display and the anatomical model and the virtual model are adjusted. The position of both anatomical and virtual models is adjusted by using the location sensor interactively. Figure 5 shows how a fetal model is displayed when it has been changed (before and after change). In this case, the anatomical model of complete breech presentation is selected.

Figure 6 shows the dialog for the selection of an anatomical model of fetus (normal one-finger dilatation has been selected) and control displays of each model (i.e. models of the mother's skin,
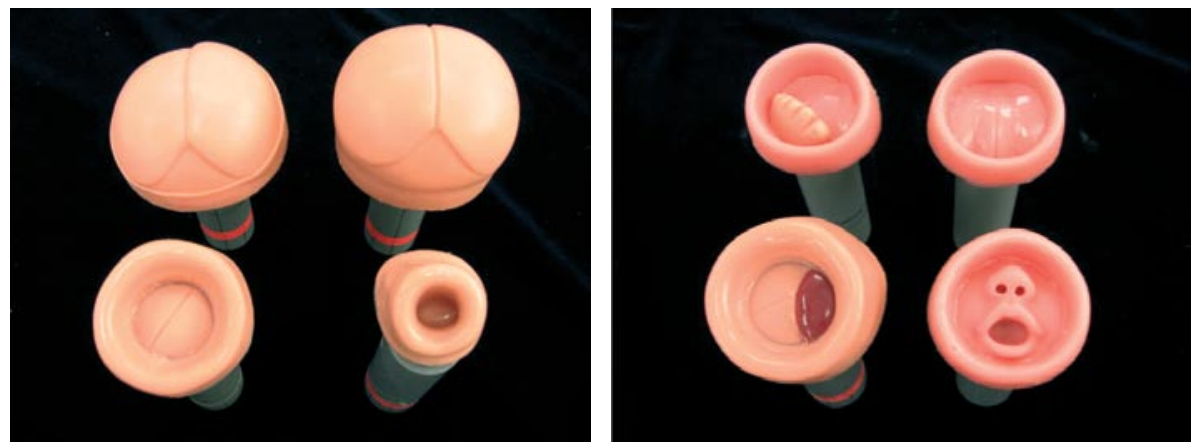

Figure 4: Anatomical models of fetus (left: normal cases, right: abnormal cases).
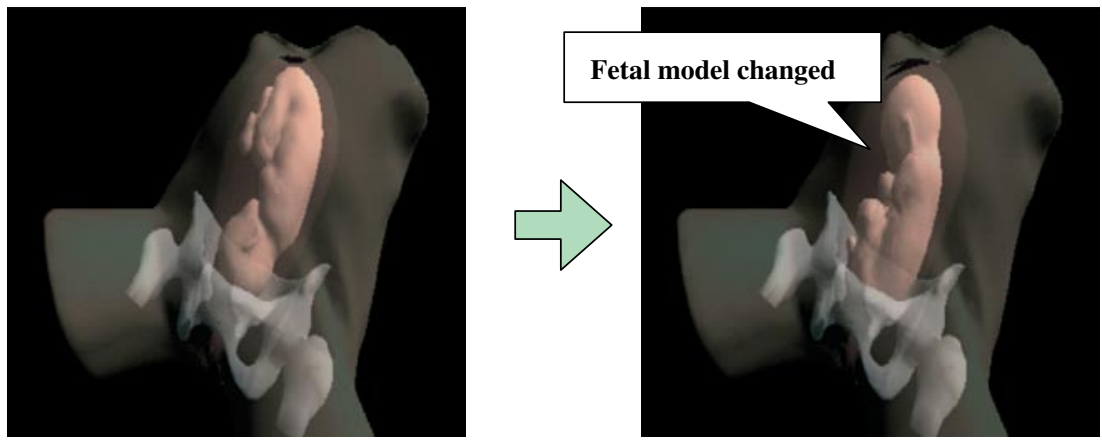

Figure 5: Change of a fetal model. 


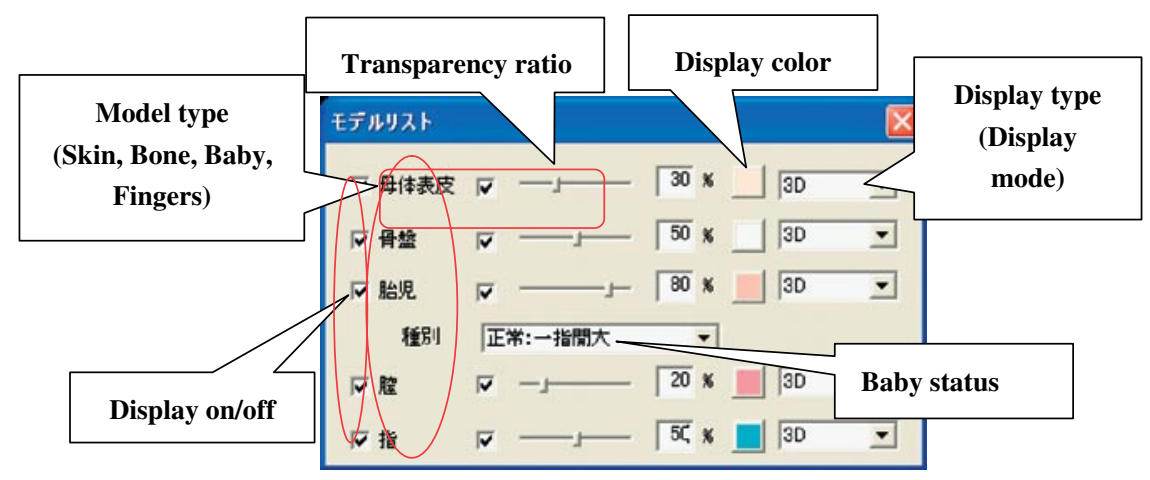

Figure 6: Fetal model selection dialog and display function.
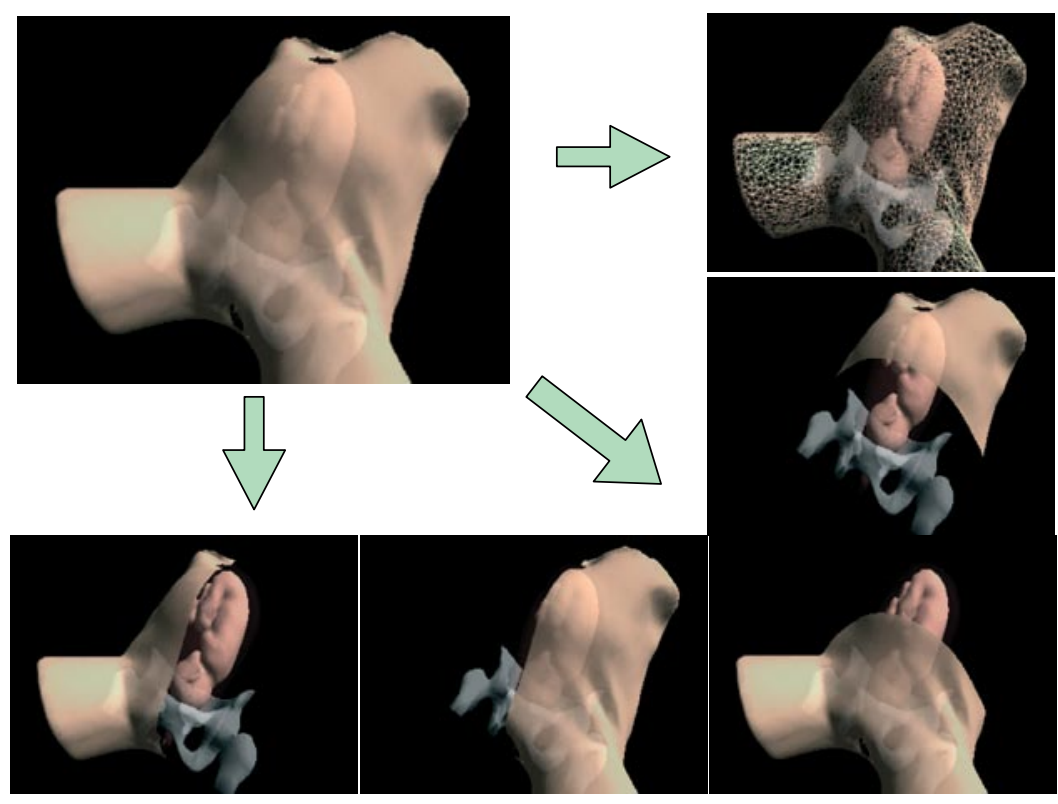

Figure 7: Display function of the internal examination training system.

pelvis, vagina, and fingers). The display control allows the selection of the constitutive models to display, the mode of display (i.e. wireframe (display with lines) or shading), transparency, and the display color. The system can also display internal views with 4 cross-sectional images (left, right, front, and back views). Figure 7 shows the example of the display control function. Normal and abnormal conditions of different fetuses can be incorporated into the system by performing the following actions: (1) creating an anatomical model of the fetus, (2) measuring or modeling the anatomical model to create a virtual model, and (3) registering the new anatomical and virtual models into the system. The positions of the anatomical and virtual models can be adjusted automatically simply by indicating the zero point of a scale called 'station' with an index finger pointer on the screen at the first application start-up.

\section{FETAL PRESENTATION}

Fetal presentation refers to the orientation of the fetus in the uterus and is classified as 'longitudinal presentation', 'transverse presentation', or 'oblique presentation'. Longitudinal presentation is 
further divided based on the position of the fetal head into the 'head presentation' (i.e. the fetal head is facing down) and 'pelvic presentation' (i.e. the fetal pelvis is facing down; also known as the breech position). All fetal presentations other than head position are considered abnormal. It is known that less than 5\% of fetuses are delivered in breech position. Delivery of fetuses in breech position carries a higher risk of experiencing difficulty in pushing out the head and causing compression of the umbilical cord than does delivery of fetuses with their head facing down (i.e. head presentation).

Breech position is roughly divided into frank breech presentation, full breech presentation, knee presentation, and foot presentation. The fetal presentation in which fetal feet are facing up and the hips facing down is referred to as the frank breech presentation, that in which both feet are facing down is referred to as complete breech presentation, and that in which only one foot is facing up is referred to as incomplete breech presentation. 'Knee presentation' is the condition in which the fetal knee is flexed and facing down during delivery (i.e. the fetus descends with its knee presenting first), and is further divided into 'complete knee presentation' (i.e. both knees facing down) and 'incomplete knee presentation' (i.e. only one knee facing down). 'Foot presentation' is the condition in which the fetus descends with its extended legs presenting first during delivery. Some breech babies can be delivered naturally through the vaginal canal if they are in frank breech presentation or full breech presentation. Breech babies in knee or foot presentation have less chance to be delivered naturally and are usually delivered by Caesarian section (cutting) [12].

\section{OPERATION OF INTERNAL EXAMINATION}

\subsection{Operation environment}

In the training system for internal examination, it is possible to change the settings for manipulation and display. [Displacement] sets the displacement in the case where the viewpoint is manipulated with the mouse, the keyboard or the [viewpoint] dialog. Larger values in the settings induce larger displacement of the viewpoint for the same manipulation (dragging distance for the mouse, number of keystrokes for the keyboard and number of button clicks for the dialog). [Display curved surfaces] controls the smoothness of the displayed surface of the model, where setting it to [Smooth] makes the displayed surface smooth. [Displayed surfaces] controls whether one or both surfaces of the model are displayed and is used when it is difficult to see the model in crosssectional view (Fig. 8). [Operation mode] sets the number of sensors used in operation. At present, the maximum supported number of sensors is two: one on the index finger and one on the middle finger. [Offset] adjusts the position of the displayed finger and is used for fine adjustment in cases where there is a discrepancy in the displayed position depending on where the sensor is placed on the finger. [Communication] is used to set the number of the COM port used for connection with the sensors. If the setting is changed, it is necessary to reset the sensor by clicking [Reset communication].

\subsection{Correspondence between the sensor and the virtual model}

When the application is started for the first time, it is necessary to match the coordinates of the virtual model and those of the finger displayed on the monitor. To simplify this process, a calibration scale referred to as 'station' is displayed in the virtual model, which facilitates the adjustment of the position of both the model and the displayed finger (Fig. 9). Specifically, their relative position with respect to each other can be adjusted by bringing the finger wearing the sensor to the station and pressing [Enter] on the keyboard. Figure 10 shows the coordinate system for the finger. 

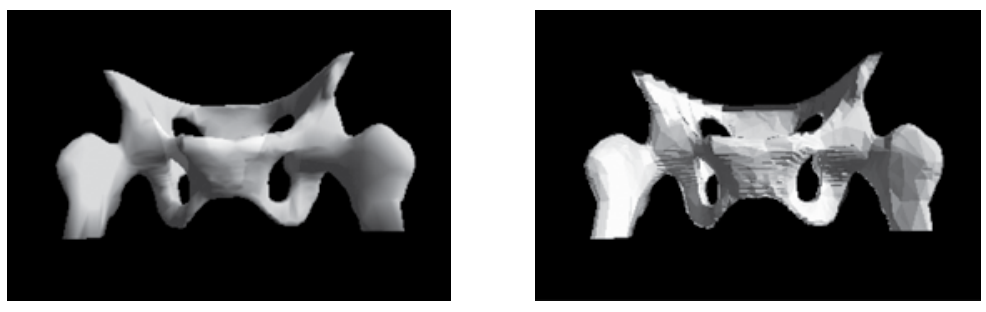

Display curved surfaces (Smooth and Flat shading display)
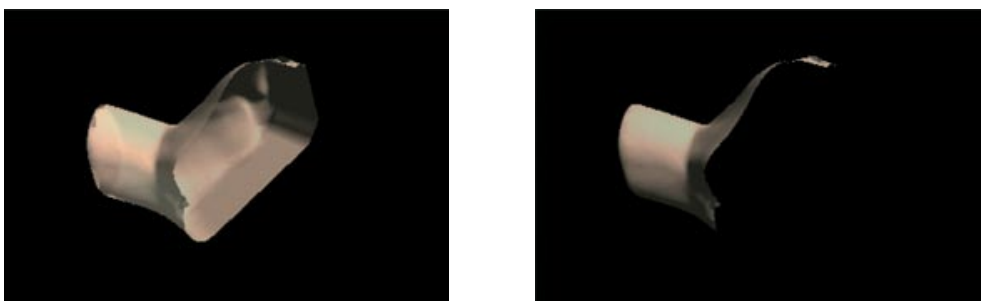

Displayed surfaces (Both surfaces and Single surface display)

Figure 8: Display mode.

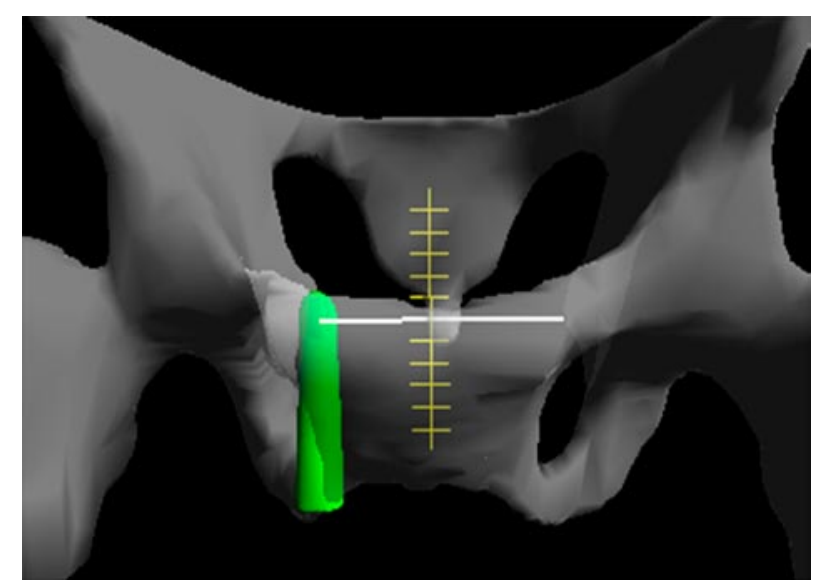

Figure 9: Correspondence between the station and the finger wearing the sensor.
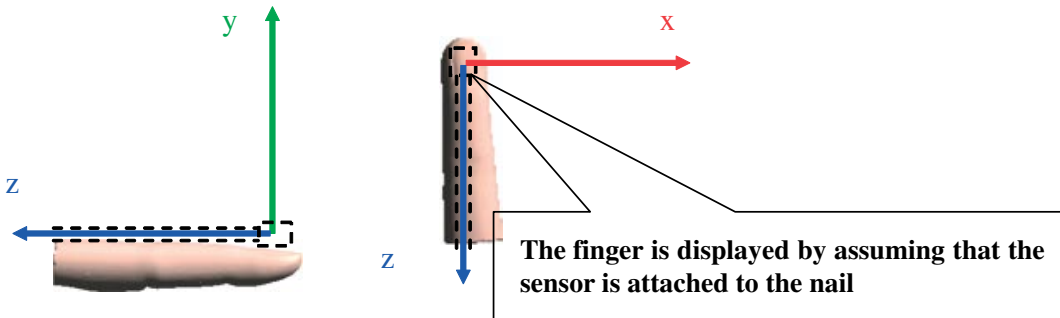

Figure 10: The coordinate system of the finger. 
(Case A) Dragging with left button pressed: rotation
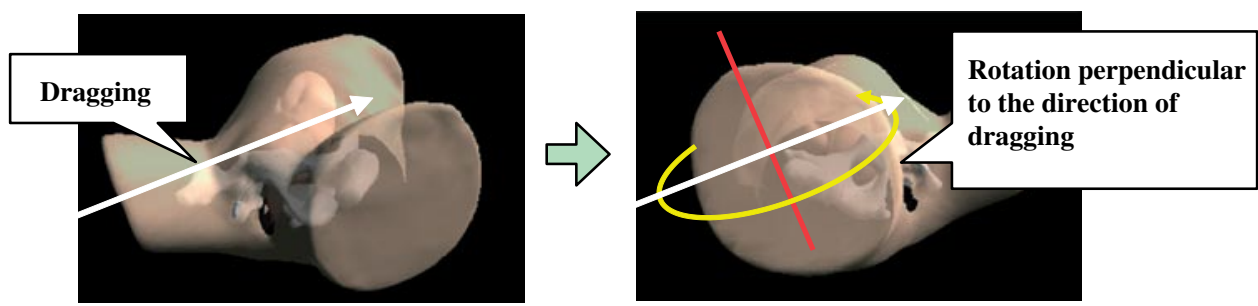

(Case B) Dragging with right button pressed: zoom in and zoom out
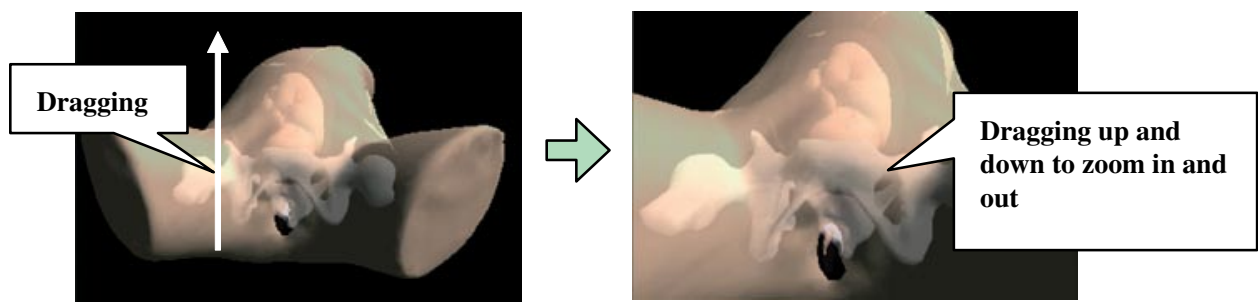

(Case C) [SHIFT] key and left button: translational motion in horizontal direction
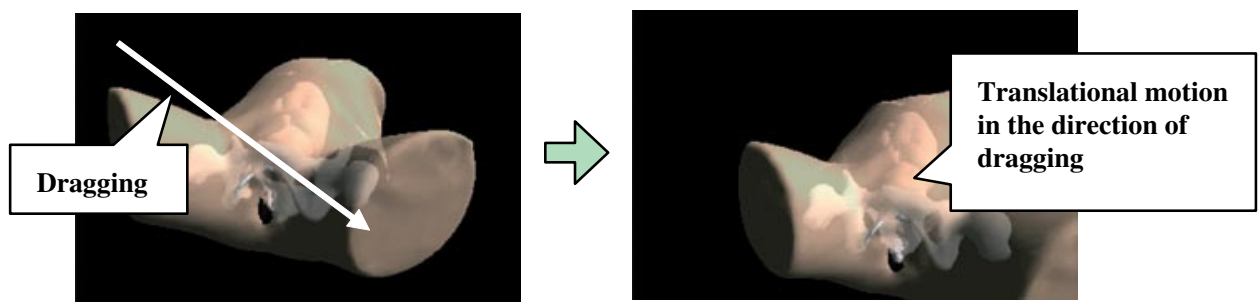

Figure 11: Operation with the mouse.

\subsection{Changing the viewpoint}

Viewing the model from different viewpoints is accomplished by dragging the cursor on the display with the mouse, where the left button controls rotation, the right button controls zooming in and out, and the model can be moved in horizontal direction by pressing the left button while holding down the [Shift] key (Fig. 11).

\section{MEASUREMENTS}

\subsection{The measurement method}

In order to measure a distance in three dimensional space, we have supported unique measurements functions using magnetic sensors. The functions are very important in order to estimate the baby status. For example, user can measure distances of a gap of the uterine ostium or baby head, a direction of baby, and so on. The teacher can also check against the measurements made by the trainees. In the training system for internal examination, distances are measured through the [Measurement] 


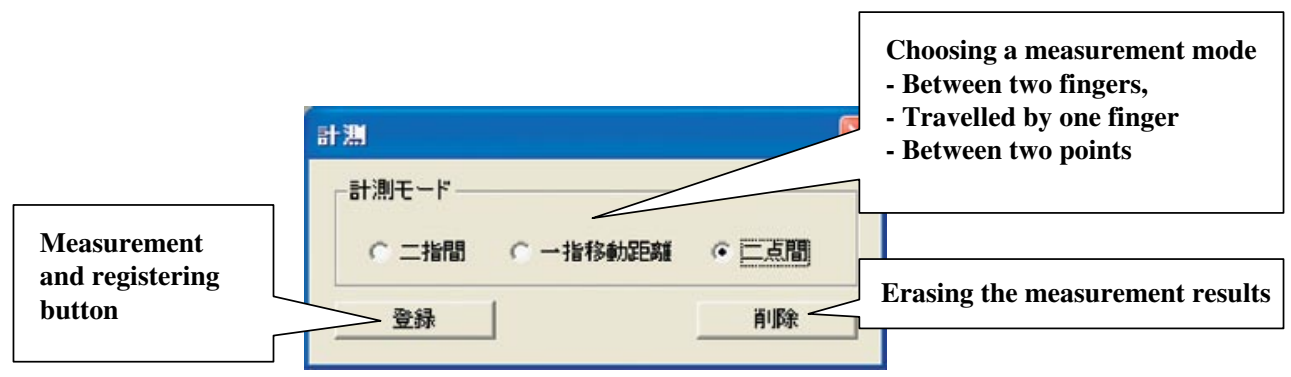

Figure 12: [Measurement] dialog.
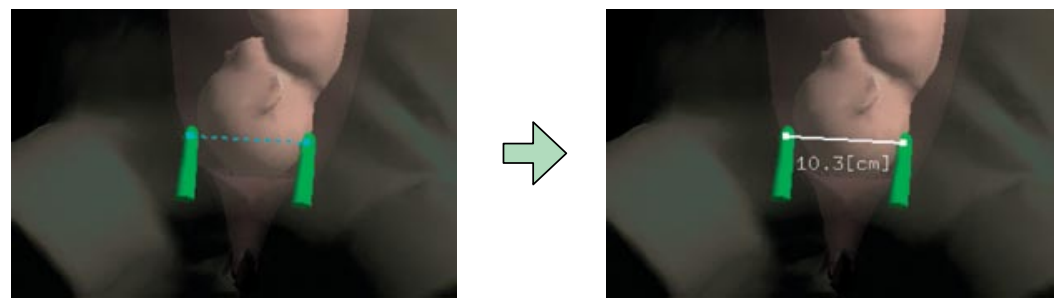

Figure 13: Measurement by between two fingers.

dialog (Fig. 12). Two measurement points are registered, and the distance between them is displayed in units of centimeter with an accuracy of one decimal place. There are three methods (measurement modes) for registering the measurement points, where the measurement method is different depending on the selected method:

- Between two fingers

- The distance travelled by one finger

- Between two points

\subsection{Between two fingers}

In this measurement mode, the measured distance is between two fingers wearing sensors. The positions of the two fingers are taken as the measurement points at the time of performing the measurement. In this case, if the mode [Between two fingers] is selected, the two fingers are moved to the region which should be measured. Measurement points are temporarily displayed on the tips of the fingers, and a dashed line is displayed between the two points. Upon pressing the [Measurement] button, the positions of the two fingers at that moment are registered as measurement points. The color of the registered measurement points is different from that of the temporary measurement points, and a solid line is displayed between them. The distance between the two points is displayed in units of centimeter with an accuracy of one decimal place (Fig. 13).

\subsection{Distance traveled by one finger}

This mode measures the distance travelled by one finger wearing a sensor. The measurement points are registered in two steps as the starting point and the ending point. Upon selecting [Distance travelled 
by one finger], the text on the [Measurement] button in the [Measurement] dialog changes to [Register], and a button for selecting the finger used for the measurement is shown. Move the finger with the selected sensor to the region to be measured. A measurement point is registered upon pressing the [Register] button and the text on the [Register] button changes to [Measurement]. Next, the finger is moved to the end point in order to perform the measurement, and a dashed line is displayed between the starting point and the finger.

After moving the finger to the end point, it is registered by pressing the [Measurement] button and the distance between the starting point and the end point is displayed (Fig. 14).

\subsection{Between two points}

Two points on the display are registered with the mouse, and the distance between them is measured. The starting point and the end point are registered in sequence, and the measurement results are displayed upon registering the end point. When the mode [Between two points] is selected, the text on the [Measurement] button in the [Measurement] dialog changes to [Register]. Upon pressing the [Register] button, the button becomes indented and waits for the registration of the measurement point. When the button is pressed again, the button restores its original shape and exits the waiting state. Move the mouse cursor to the starting point in the region to be measured. The first point is registered by clicking. Next, the mouse is moved again in order to record the end point. A dashed line is displayed between the starting point and the end point. After moving the mouse, the end point is registered by clicking and the distance between the starting point and the end point is displayed (Fig. 15).

As measurements are performed repeatedly, the results are displayed accumulatively on the display. The [Erase] button can be used for erasing the results (Fig. 16).

\section{SYSTEM EVALUATION}

We conducted a survey of eight students at our university, and made inquiries for our system, and evaluated it for understanding where the position of ischiatic thor (spinal ischium), baby head, and uterine ostium. We also checked that it was easy to understand the rotation of baby head and the open of the uterine ostium. In the lecture of an internal examination, first we teach an internal examination
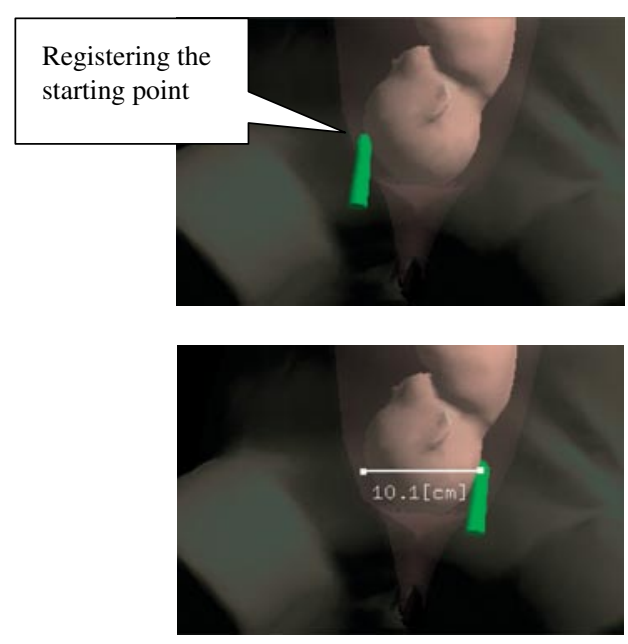
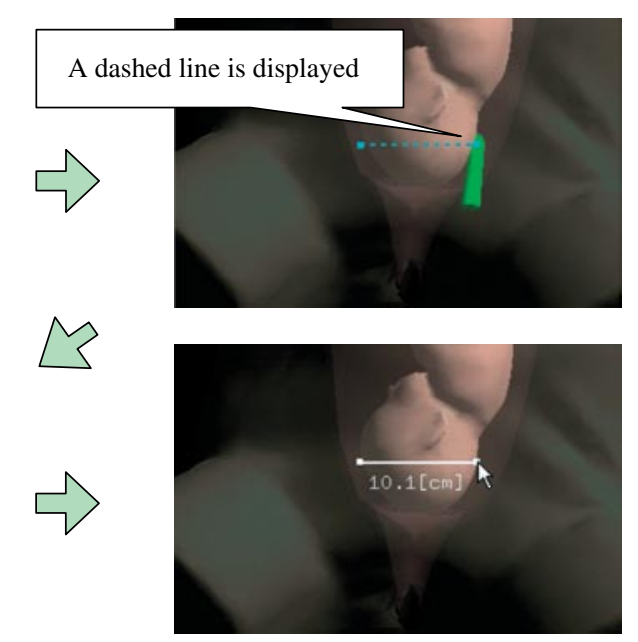

Figure 14: Measurement by distance travelled by one finger. 

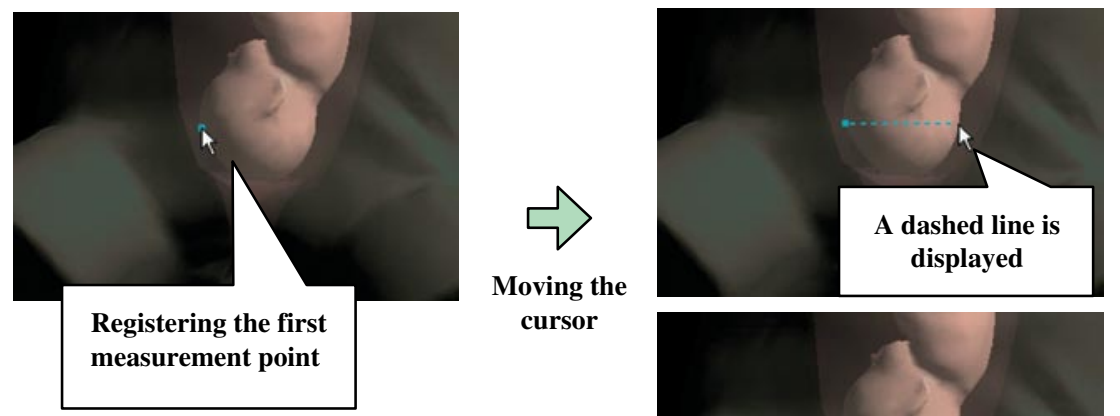
cursor

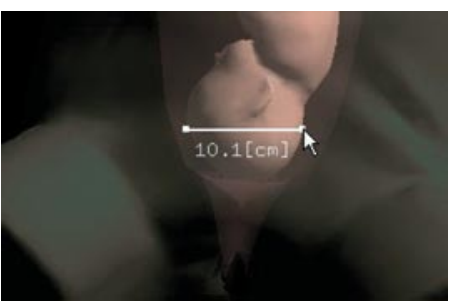

Figure 15: Measurement by between two points.
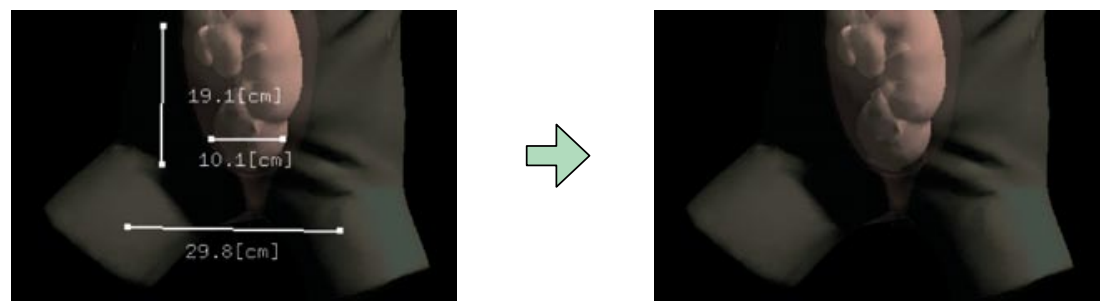

Figure 16: Erasing the measurement points.
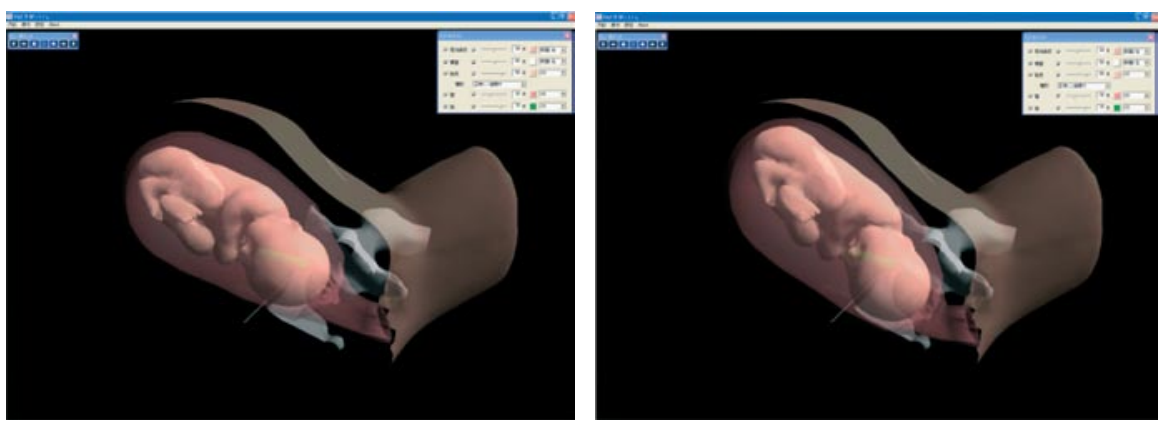

Figure 17: Fetal models of normal conditions (left: one-finger dilatation, right: two-finger dilatation).

in the classical method using a manikin only. Next, we teach them by using our system and make inquiries for our system with unregistered style. We check understanding of the students for several questions before and after looking images in our system. The result of several questions shows that the understanding of all students is improved by using our system [13]. Figures 17 and 18 show fetal models of normal conditions supported by the internal examination training system (i.e. one-finger dilatation, two-finger dilatation, type C (almost full dilatation), and full dilatation). Figures 19 and 20 show fetal models of abnormal conditions (incomplete foot presentation (frank breech presentation), complete breech presentation, placenta previa, and face presentation). 

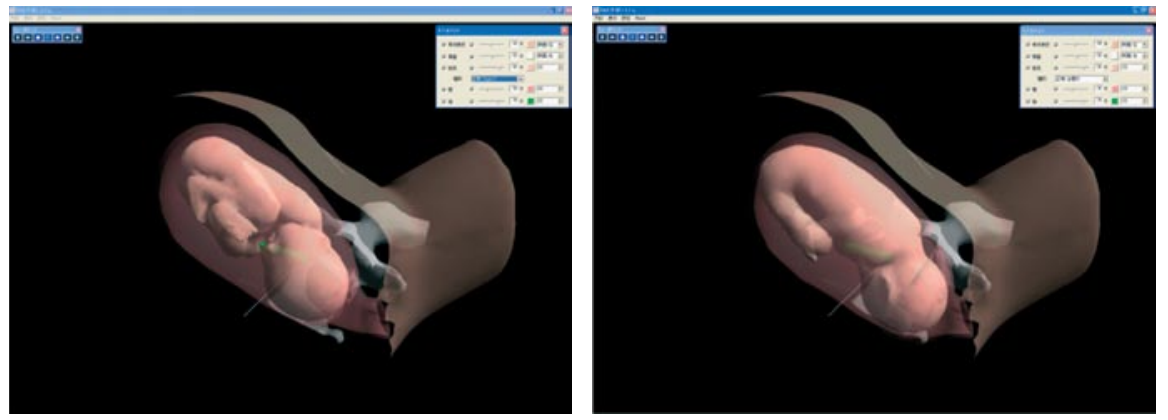

Figure 18: Fetal models of normal conditions (left: Type C, right: full dilatation).
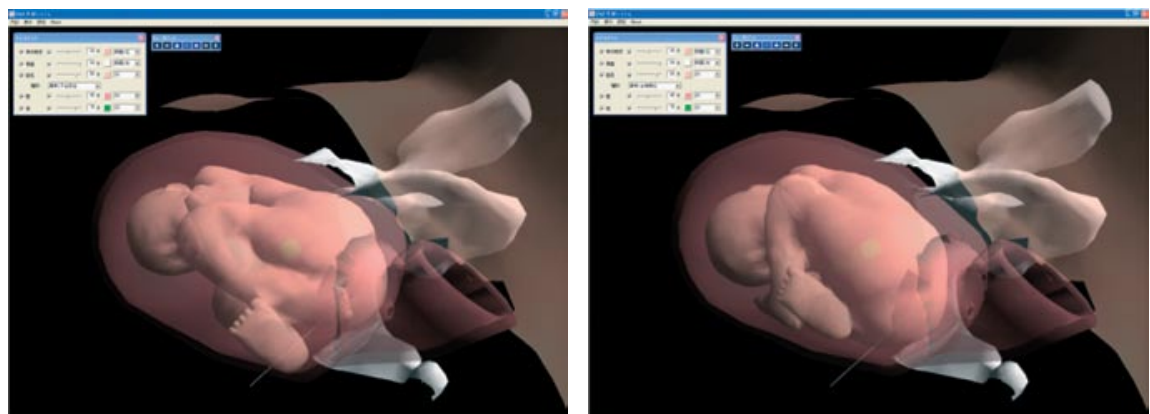

Figure 19: Fetal models of abnormal conditions (left: incomplete foot presentation, right: complete breech presentation).
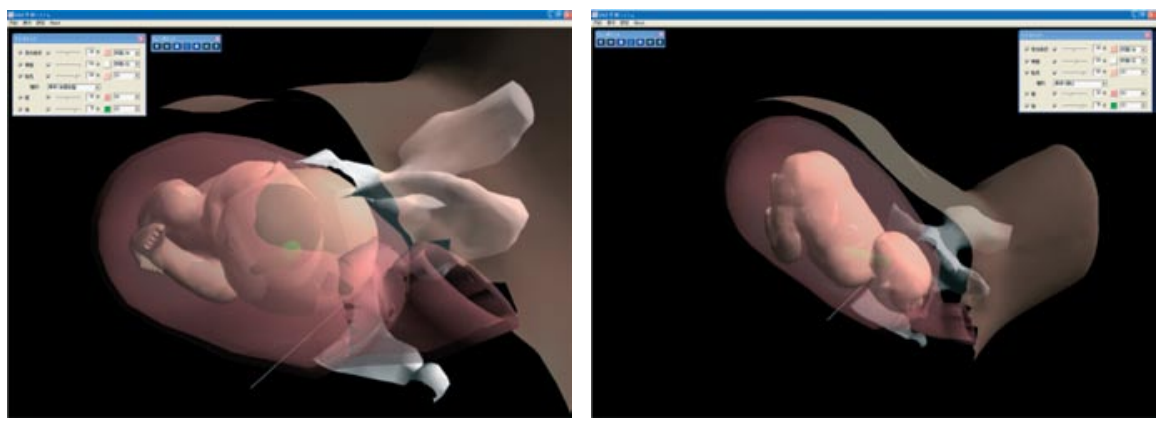

Figure 20: Fetal models of abnormal conditions (left: placenta previa, right: face presentation).

We have been evaluating the usefulness of the present technique by disseminating its use as a learning tool and presenting it at international meetings and exhibitions. At the exhibition of the 21st Japan Academy of Midwifery Scientific Meeting (Oita prefecture in Japan, March 10 and 11, 2007), we presented and demonstrated our internal examination training system and a number of people experienced the training procedure and answered a questionnaire about the usefulness of the system. Over 100 people attended our presentation, approximately 60 of whom experienced 
the training procedure and provided feedback. Most of those who experienced the training system at the exhibition of the 21st Japan Academy of Midwifery Scientific Meeting provided positive comments, although some requested improvements or the addition of functions. These requests included the addition of various abnormal labor conditions, more measurement functions (e.g. distance between two fingers and distance to the head of fetus), and an internal examination rating function for each trainee. We have satisfied one of the requests by developing 4 abnormal labor conditions (frank breech presentation, complete breech presentation, placenta previa and face presentation) and have included them in the latest training system. We have also added measurement functions using one or two fingers; the location sensors mounted on the index and middle fingers enable the acquisition of position information in space (i.e. spatial position and rotation). Using this measurement function, we can precisely measure spatial distances estimated on the basis of the distance between the two fingers or the measurer's experience.

\section{CONCLUSION}

We have developed an internal examination training system that supports the learning of normal and abnormal labor conditions. The system provides a unique learning experience that cannot be gained in daily practice. The use of the system in teaching practice will help to nurture competent obstetricians and midwives in a short period. On the basis of specialist' feedback, we included both 4 normal and 4 abnormal fetal models in the training system. The availability of additional abnormal fetal models will make the system more relevant to actual clinical situations. The system supports several measurement functions using one or two fingers to which magnetic sensors are attached. Currently, we have developed the more real finger model, which represents finger bending by using 5DT Data Glove [14]. The glove itself does not contain any magnetic parts, and provides finger bending information by communicating with a control box via optical fiber. In order to construct the finger skin model, a human hand was captured by Magnetic Resonance Imaging device. We believe that the realistic finger model improves understanding of internal examination. Recently, Ascension Technology Corp. has announced the fourth generation of magnetic sensor, which is called trackSTAR [15-17]. The trackSTAR is the latest addition of the Ascension Technology Corp. magnetic sensors, and the sensor size for full six degrees-of-freedom tracking is just $1.5 \mathrm{~mm}$ in diameter. In order to utilize the device, we have incorporated the device drivers into our system. The differences between the previous magnetic sensors and the trackSTAR are the fast and dynamic tracking (240 to 420 measurements per second), compact transmitter, no external power supply, the small size of control box, and the support for the interchangeable sensor sizes. In comparison with miniBIRD, the trackSTAR has 4 ports for sensors, and can use by 4 sensors simultaneously. Therefore, our system configuration becomes more compact and simple. In future studies, we aim to perform more detailed examinations of abnormal labor conditions, and a useful internal examination rating feature for each trainee should be supported in our system. Although the present system was developed specifically for internal examination of the fetus, it can also be applied for such purposes as medical training and preoperative planning for other parts of the body (e.g. thorax and abdomen) by using different anatomical and virtual models.

\section{ACKNOWLEDGEMENTS}

This research was supported in part by Grants-in-Aid for Scientific Research (20500425), and grants aid for scientific research of Iwate Prefectural University (a basis research fund and cooperation research fund (2002)). We would like to thank the reviewers for helpful comments. 


\section{REFERENCES}

[1] Ishii, T., Doi, A., Katamachi, K., Noguchi, K., \& Uno, H., Medical Training Device, Japan Patent Application No. 2005-032614, Reference No. 4-1130, Receipt No. 50500225450, Patent Applicant: KOKEN Co., Ltd., 2004.

[2] Ishii, T., Doi, A., Katamachi, K., Noguchi, K., \& Uno, H., Medical Training Device, International patent application (European Patent Office), Reference No. GP05-1033PCT, Receipt No. 50600235406, Application No. Notification: PCT/JP2006/30219, Patent Applicant: KOKEN Co., Ltd., 2005.

[3] Doi, A., Matsui, K., Katamachi, K., Noguchi, K., Ishii, T., Uno, H., A computer assisted medical training system for checking status of delivery by using virtual reality technique and physical models, CARS2007, pp. 156, 2007.

[4] Carla M. Pugh, et al., The E-Pelvis: A Pelvic Examination Simulator, SUMMIT (Stanford University Medical Media \& Information Technologies) Project Overview, On-line http://summit. stanford.edu/publications/tear_index.htm

[5] SUMMIT (Stanford University Medical Media \& Information Technologies, e-Pelvis, On-line http://summit.stanford.edu/research/epelvis.html

[6] AISR of Thomas Jefferson University, Jefferon:Technology in the Classroom, On-line http:// jeffline.jefferson.edu/AISR/Forum/04/07/pdfs/ClassTech.pdf

[7] MC Medical Shuppan, 3D CG Peripartum Diagnosis/Delivery Assistance Training System, On-line http://www.medica.co.jp/3d-bunben/\#top

[8] [z]Buyamag Inc., SIMone Childbirth Birthing Simulator/Manikin, Online http://www. buyamag.com/gynecological_models.php

[9] Doi, A., Noguchi, K., Katamachi, K., Ishii, T., Uno, H., Mega, Y., \& Matsui, K., An internal examination training system supporting abnormal labor conditions. Biomed 2009, Eighth International Conference on Modelling in Medicine and Biology, 26-28 May 2009.

[10] Mindflus, miniBIRD 500\&800, On-line http://www.mindflux.com.au/products/ascension/ minibird-500-800.html

[11] 5DT Fifth Dimension Technology, miniBIRD Model 800 and Model 500, On-line http:// www.5dt.com/downloads/3rdparty/minibird.pdf

[12] Yajima, S., Nakano, H., \& Taketani, Y., NEW Gynecologic Sciences, Nankodo; 2nd edn, 2004.

[13] Noguchi, K., \& Ishii, T., The development of teaching material for pelvic examination by combination of virtual reality techniques and physical model. Journal of Japan Academy of Midwifery, 20(3), pp. 134, 2007.

[14] 5DT Fifth Dimension Technology, 5DT Data Glove 5 MRI/5DT Data Glove 16 MRI, On-line http://www.5dt.com/index.html

[15] Ascension Technology Corp., Tracking 3D worlds, On-line http://www.ascension-tech.com/

[16] Ascension Technology Corp., 3D guidance trackSTAR, On-line http://www.ascension-tech. com/realtime/RTtrakSTAR.php

[17] Inition Corp., 3D Guidance driveBAY, On-line http://www.inition.com.au/inition/pdf/mocap_ ascension_drivebay.pdf 